\title{
Identification of the Early Actin-Rearrangement-Inducing Factor Gene, arif-1, from Autographa californica Multicapsid Nuclear Polyhedrosis Virus
}

\author{
RENZA RONCARATI AND DAGMAR KNEBEL-MÖRSDORF* \\ Institute of Genetics, University of Cologne, D-50931 Cologne, Germany
}

Received 28 March 1997/Accepted 1 July 1997

\begin{abstract}
In response to Autographa californica multicapsid nuclear polyhedrosis virus (AcMNPV) infection, a sequential rearrangement of the actin cytoskeleton occurs. Previous studies suggest that the penetration of nucleocapsids induces early actin cables followed by further changes of the actin cytoskeleton which depend on early viral gene expression. By transfection of a plasmid library into Trichoplusia ni TN-368 cells, we have identified an early viral gene, designated arif-1, that is able to induce actin rearrangement. The determination of the nucleotide sequence of arif-1 revealed one open reading frame potentially encoding a gene product of $45 \mathrm{kDa}$ with no significant sequence homology to known proteins. After expression of arif-1 in transfected cells, the induced actin rearrangement, visualized by fluorescence microscopy, was comparable to the changes of the actin cytoskeleton at 3 to $7 \mathrm{~h}$ postinfection. These changes are based on early viral gene expression during the infection cycle. A causal link between arif-1 expression and actin rearrangement in infected cells is suggested by infection studies with the AcMNPV/Spodoptera frugiperda MNPV hybrid, which carries a deletion in the arif-1 gene. In transfection experiments the presence of the known viral transactivator IE1 was required in addition to ARIF-1 to induce actin rearrangement. IE1 was needed for promoter activation of the arif-1 gene, since arif-1 expression under the control of the early pe38 promoter was sufficient to induce actin rearrangement in transfected cells. Primer extension analyses showed that the arif-1 gene is transcribed only during the early phase of AcMNPV infection in T. ni TN-368 cells. There was a delay of about $1 \mathrm{~h}$ compared to $i e 1$ transcription, which is in agreement with the assumption that IE1 transactivates the arif-1 promoter during infection.
\end{abstract}

For a number of viruses, alteration of the actin cytoskeleton in response to viral infection has been observed (for a review, see reference 26), although the functional role still remains unclear. Recent studies on vaccinia virus support the view that viruses can use the actin cytoskeleton of the host cell to move to the cell surface to contact neighboring cells $(9,10)$. An alternative usage of the actin cytoskeleton might be accomplished by the baculovirus Autographa californica multicapsid nuclear polyhedrosis virus (AcMNPV). It is a large DNA virus, with a genome of $133,894 \mathrm{bp}(1)$, which replicates in the cell nucleus. During viral infection, two different infectious particles which are essential to complete the infection cycle in the larvae are formed (for a review, see reference 3). In infected insect cells AcMNPV gene expression, which is temporally ordered in a cascade, results in a characteristic cytopathic effect. The infected cells round up, the nuclei are enlarged, and the virogenic stroma appears in the center of the nuclei (for a review, see reference 31). The change of the cell shape correlates with a drastic alteration of the microtubule network (33). In addition, the actin cytoskeleton is rearranged in a number of distinct steps during the course of infection. Three stages of actin rearrangement have been distinguished in infected Spodoptera frugiperda cells (6). After penetration of budded viruses by adsorptive endocytosis, actin cables appear, followed by the formation of F-actin aggregates at the ventral surface of the cells. Later during nucleocapsid morphogenesis, microfilaments are present in the nucleus within the ring zone surrounding the virogenic stroma. When infected cells are treated

\footnotetext{
* Corresponding author. Mailing address: Institut für Genetik, Universität zu Köln, Weyertal 121, 50931 Köln, Germany. Phone: 49-2214703528. Fax: 49-221-4705163. E-mail: moed@scan.genetik.uni-koeln .de.
}

with cytochalasin $\mathrm{D}$, which interferes with actin polymerization and function, colocalization of F-actin within the ring zone does not appear and nucleocapsid morphogenesis is inhibited $(24,30,32,34)$. Therefore, nuclear F-actin is thought to play a key role in nucleocapsid assembly.

The function of actin cables and aggregates which are present in the cytoplasm during the early phase of infection is currently not well understood. Induction of early actin cables is independent of virus adsorption and uptake into the endocytic vesicles but correlates with the release of nucleocapsids from the endosomes (7). In unfixed cells, nucleocapsids are frequently observed to be associated with one end of the actin cables, which might indicate the putative role of the cables in the movement of the nucleocapsids to the nucleus (7). Recent studies have described two actin-targeting activities which are associated with budded viruses and may induce actin cables (20). In contrast to the formation of actin cables, the appearance of actin aggregates is thought to depend on early viral gene expression (6). Aggregates of actin on the ventral site of infected cells cannot be detected when protein synthesis is blocked with cycloheximide (6).

The objective of this study was to demonstrate that early viral genes are involved in the induction of actin rearrangement and to identify the corresponding gene(s). An early gene which we designated arif-1 (for actin-rearrangement-inducing factor 1) was identified by screening a plasmid library in transient-expression assays. Expression of arif-1 alone led to actin rearrangement which was comparable to the second step of actin rearrangement during infection but differed in appearance from the initial formation of actin cables after penetration of nucleocapsids. Therefore, it is likely that ARIF-1 has a functional role during the infection cycle. 

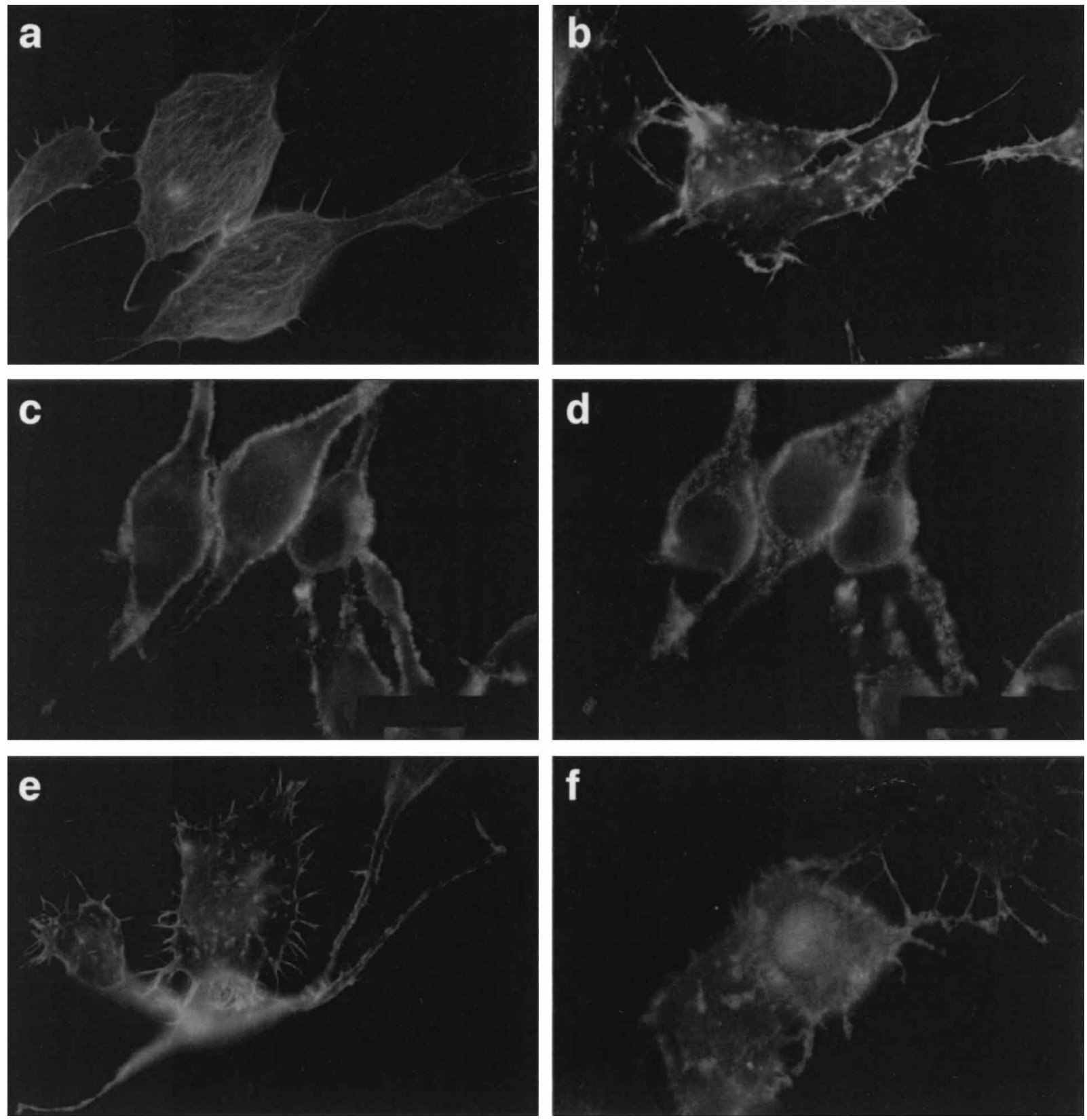

FIG. 1. Organization of the actin cytoskeleton in uninfected and AcMNPV-infected TN-368 cells and after transfection of the genomic AcMNPV DNA. Uninfected cells (a) or cells infected with AcMNPV (b, c, d, and e) were fixed in paraformaldehyde at 2 (b), and 6 (c, d) h p.i. The same cells were photographed at a ventral (c) or a medial (d) plane of focus. (e) Cycloheximide-treated cells were fixed $4 \mathrm{~h}$ p.i. (f) Cells treated with aphidicolin were transfected with AcMNPV DNA, fixed at $40 \mathrm{~h}$ after transfection, and visualized by staining with anti-PE38 antiserum and FITC-conjugated anti-rabbit IgG. The actin cytoskeleton was labeled with TRITCconjugated phalloidin.

\section{MATERIALS AND METHODS}

Cells, virus, and inhibitor treatment. Trichoplusia ni TN-368 cells (16) were grown as monolayer cultures in TC100 medium (12) supplemented with $10 \%$ fetal calf serum. Infection with the AcMNPV plaque isolate E (28) was performed at a multiplicity of infection of 10 PFU per cell. Time zero was defined as the time when the AcMNPV inoculum was added to the cells. Cells were treated with $1 \mathrm{mg}$ of cycloheximide (Sigma) per ml for $2 \mathrm{~h}$ prior to AcMNPV infection. After adsorption of the virus inoculum for $1 \mathrm{~h}$, fresh medium containing cycloheximide $(1 \mathrm{mg} / \mathrm{ml})$ was added. Two hours prior to transfection with plasmid DNA, the cells were treated with aphidicolin $(5 \mathrm{mg} / \mathrm{ml}$; Sigma).

Plasmid constructions. A HindIII genomic library was constructed by digesting the AcMNPV genome with HindIII and cloning the resulting fragments into plasmid pUC18 or pBR322 (18a). Plasmids pClaI-ie1 (23) and pPE-HR1 (19) have been described previously. Subclones of the fragment HindIII-D, corre- sponding to nucleotides (nt) 9053 to 19054 in the published sequence of AcMNPV (1), were generated as follows: the plasmid pHindIII-D was digested with Bam HI, generating a 3.7-kb fragment and a 5.9-kb vector-containing fragment which was religated and designated pHB5.9. The 3.7-kb fragment was inserted into the Bam HI site of pBluescript $\mathrm{KS}(+)$, generating plasmid pBH3.7. The constructs pBP2.1 and pPstI-O were built by isolating a BamHI/Pst I $(2.1-\mathrm{kb})$ fragment and the Pst $\mathrm{I}-\mathrm{O}(1.6-\mathrm{kb})$ fragment from $\mathrm{pBH} 3.7$ and inserting them into pBluescript $\mathrm{KS}(+)$ digested with Bam $\mathrm{HI} /$ Pst I or Pst I (see Fig. 2).

The plasmid pARIF-1 was generated by insertion of a 1,751-bp PCR-amplified fragment into the HindIII and BamHI sites of pBluescript $\mathrm{KS}(+)$. The PCR fragment encompasses the arif- 1 open reading frame (ORF) and $3^{\prime}$ and $5^{\prime}$ flanking sequences and was amplified from the plasmid $\mathrm{pBH} 3.7$ by using the primers 5'-TTTTAAGCTTCTTGTTCAGTTCTAATTATTG-3' and 5'-TTTT GGATCCGCACCGACATGCATTTAATCG-3'. After digestion of pARIF-1, 


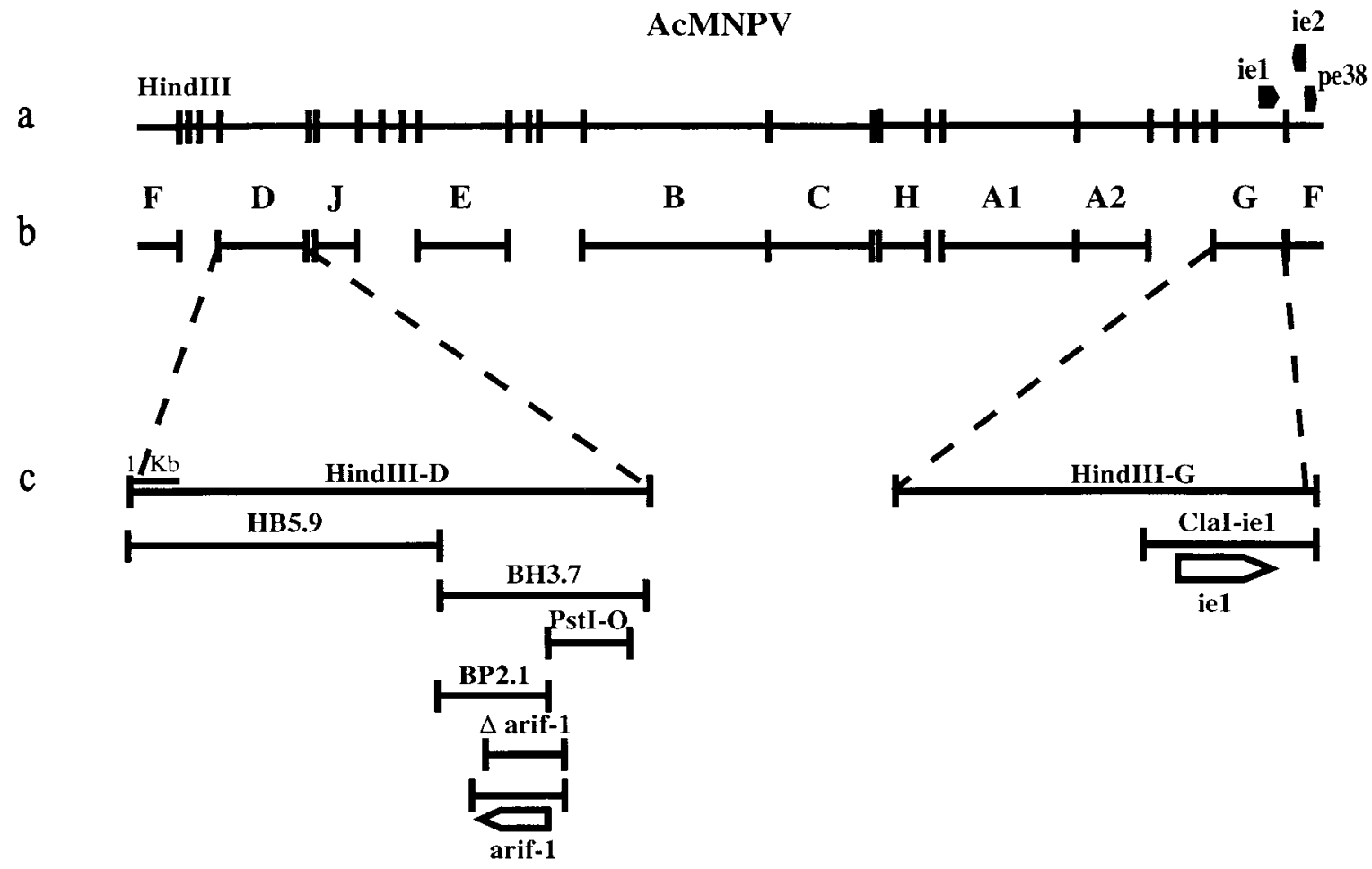

FIG. 2. AcMNPV map and cloned viral fragments. (a) Linearized AcMNPV genome with the HindIII restriction sites. The ie1, ie2, and pe38 ORFs are indicated by arrows. (b) Cloned HindIII fragments representing the plasmid library. (c) Subclones of the HindIII-D and HindIII-G fragments as described in Materials and Methods. The arif-1 and ie1 ORFs are represented by open arrows.

the $X b a \mathrm{I}$ fragment was inserted into pBluescript $\mathrm{KS}(+)$ digested with $X b a \mathrm{I}$. The resulting plasmid, $\mathrm{p} \Delta \mathrm{ARIF}-1$, lacks 164 codons at the $\mathrm{C}$ terminus and the $3^{\prime}$ end of the arif-1 gene. The construct pFHC-PE38/ARIF-1 contains the arif-1 ORF under the control of the pe38 promoter and was built as follows. The plasmid pFHC-HR1 (19) was partially digested with StyI, blunt ended by fill-in reaction with Klenow polymerase, and cleaved with $C l a$ I. The vector fragment containing the complete $i e 2$ gene was ligated with the $C l a \mathrm{I} / P s t \mathrm{I}$ (blunt-ended) fragment of pARIF-1 and an oligonucleotide which was flanked by a blunt end and a Pst I site. Therefore, in plasmid pFHC-PE38/ARIF-1 the ATG start codon of arif-1 is replaced by that of pe38.

Determination of the nucleotide sequences. The sequence of the plasmid pARIF-1 was determined by using the dideoxy chain termination method (27) and appropriate primers for PCR. The nucleotide sequences were computer analyzed in an Applied Biosystems 373A DNA sequencer. In addition, the 3' part of $\mathrm{pBP} 2.1$ and the $5^{\prime}$ part of pPst-O were sequenced.

Transcriptional mapping. For primer extension analysis, cytoplasmic RNA $(10 \mu \mathrm{g})$ was annealed to an arif-1-specific 30-base primer (5'-CGTA TCTGCTGTCTATCACTCCCATTATAG-3'), an ie1-specific 31-base primer (5'-GGGTTGTTTATCACAAAACTCTGAATAGCTG-3'), and a pe38-specific primer (19) as described earlier (2).

Transfection experiments. TN-368 cells were seeded on coverslips $\left(10^{5}\right.$ cells per coverslip) in Grace's medium (Gibco) without serum supplementation. At $2 \mathrm{~h}$ after seeding, cells were transfected with $1 \mu \mathrm{g}$ of each plasmid via liposomemediated transfection. The DNA $(0.1 \mathrm{mg} / \mathrm{ml})$ was mixed with a liposome suspension (DOTAP; Boehringer) according to the instructions of the manufacture and applied to the cells after incubation for $15 \mathrm{~min}$ at room temperature. At $6 \mathrm{~h}$ after transfection, the medium was replaced by TC100 medium supplemented with $10 \%$ fetal calf serum. For immunofluorescence the cells were processed $40 \mathrm{~h}$ after transfection.

Polyclonal antibodies. The production of polyclonal antisera directed against PE38 and IE2 was described previously (19). The polyclonal anti-IE1 is directed against the $\mathrm{N}$ terminus of IE1, which was overexpressed as a fusion protein with dihydrofolate reductase in bacteria (16a).

Immunocytochemistry. TN-368 cells which were grown on coverslips were rinsed with phosphate-buffered saline (PBS), fixed in $2 \%$ paraformaldehyde for $15 \mathrm{~min}$ at room temperature, and subsequently permeabilized by incubation with $0.4 \%$ Triton X-100 for $4 \mathrm{~min}$. Cells on coverslips were floated for $1 \mathrm{~h}$ on $30 \mu \mathrm{l}$ of primary antibodies at a 1:1,000 dilution for anti-IE2 and anti-PE38 antisera and a 1:500 dilution for anti-IE1 antiserum in a PBS buffer containing 2\% bovine serum albumin. The cells were washed three times in PBS and incubated for 45 min with fluorescein isothiocyanate (FITC)-conjugated anti-rabbit immunoglob- ulin G (IgG) (Nordic Immunological). Staining of F-actin was achieved by using tetramethylrhodamine isothiocyanate (TRITC)-conjugated phalloidin at a concentration of $3 \times 10^{-7} \mathrm{M}$ as described previously (6). Cells on coverslips were rinsed several times with PBS and mounted on a drop of 1,4diazabicyclo[2.2.2] octane. Specimens were viewed with a Zeiss Axioscop H microscope.

\section{RESULTS AND DISCUSSION}

Early actin rearrangement in AcMNPV-infected TN-368 cells. Changes in the actin cytoskeleton were analyzed during the early phase of AcMNPV infection in TN-368 cells. In contrast to previous studies, which were performed with $S$. frugiperda cells (6), we chose the insect cell line TN-368 because of its higher susceptibility to AcMNPV infection $(11,22)$ and because of its morphology. Adherent TN-368 cells are mostly oval with two or more protoplasmic extensions and are larger than the spherical $S$. frugiperda cells.

Microfilaments which were stained with TRITC-conjugated phalloidin formed a fine homogeneous network in uninfected cells (Fig. 1a). At 2 and $6 \mathrm{~h}$ postinfection (p.i.) the actin cytoskeleton changed dramatically, although the cell shape had not yet altered. At $2 \mathrm{~h}$ p.i. actin cables, which mostly were localized at the cell surface and extended into the cytoplasm, appeared (Fig. 1b). A second stage of actin rearrangement was observed at 3 to $7 \mathrm{~h}$ p.i., during which actin cables became more prominent and accumulated at the cell borders (Fig. 1c and d). When cycloheximide was added prior to infection to inhibit viral and cellular protein synthesis, only the first stage of actin rearrangement took place (Fig. 1e). These observations are in agreement with previous results which were obtained with AcMNPV-infected $S$. frugiperda cells (6).

Early actin rearrangement after transfection of viral DNA or cloned viral DNA fragments. To strengthen the view that 

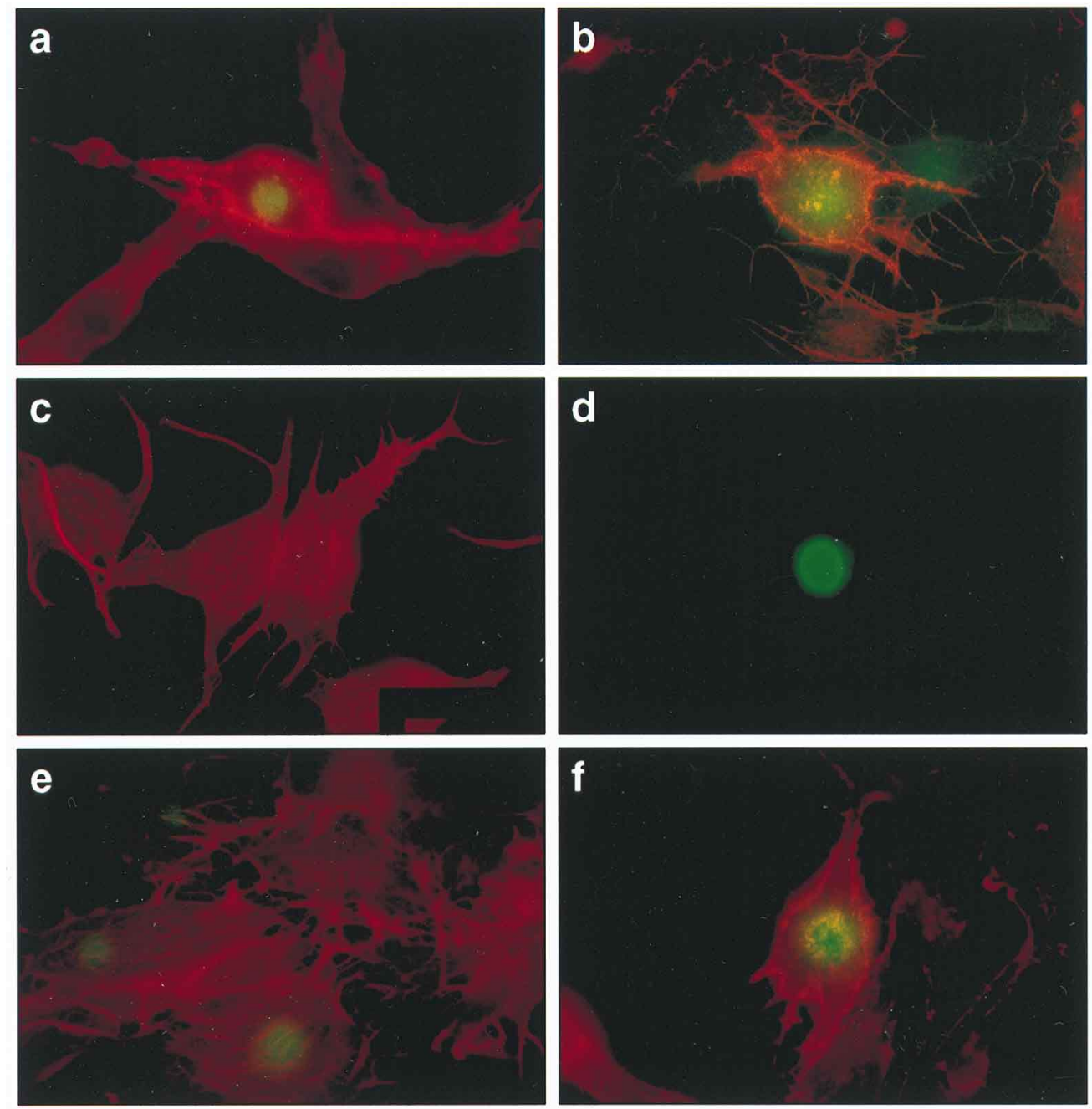

FIG. 3. Organization of the actin cytoskeleton after transfection with plasmid DNA. TN-368 cells were transfected with the plasmid library (a); with plasmids pHindIII-D, pHindIII-G, and pPE-HR1 (b); with plasmids pPst-O, pBP2.1, and pCla-ie1 (c and d); with plasmid pHindIII-G (e); and with plasmids pARIF-1 and pClaI-ie1 (f). The cells were fixed in paraformaldehyde $40 \mathrm{~h}$ after transfection. Transfected cells were visualized by staining with anti-PE38 (a and b) or anti-IE1 (d, $\mathrm{e}$, and f) antisera and FITC-conjugated anti-rabbit IgG. The actin cytoskeleton was labeled with TRITC-conjugated phalloidin. After double labeling, the cells were viewed through different filters (c and d); labeled microfilaments are shown (c), and the transfected cell was visualized with anti-IE1 antiserum, which is localized in the nucleus (d).

early viral gene products are involved in the second stage of actin rearrangement that is observed at 3 to $7 \mathrm{~h}$ p.i. and is independent of virus replication (6), AcMNPV DNA was transfected into TN-368 cells. Prior to transfection, the cells were treated with aphidicolin to block viral replication (25), and they were fixed at $40 \mathrm{~h}$ after transfection. In addition to TRITC-conjugated phalloidin, the cells were stained with polyclonal antibodies directed against the early viral gene product PE38 or IE2 (19) to detect the transfected cells. In most transfected cells, actin rearrangement (Fig. 1f) which was comparable to that in infected cells at 3 to $7 \mathrm{~h}$ p.i. was observed. As a control, transfected cells were kept as long as 5 days in culture to test for the formation of polyhedra. Since no polyhedra were visible, viral replication was efficiently blocked. These results indicate that early viral gene products indeed are able to cause changes in the actin cytoskeleton irrespective of the presence of nucleocapsids. To exclude any influence of the transfection procedure on the induction of actin rearrangement, we performed mock transfection experiments or trans- fected unrelated vectors, which never led to actin rearrangement.

In order to identify the early gene(s) that is responsible for the induction of actin rearrangement, TN-368 cells were transfected with a library of 10 cloned HindIII fragments covering nearly $80 \%$ of the AcMNPV genome (Fig. 2). Transfected cells, which were highlighted by staining with antibodies against PE38, showed actin rearrangement (Fig. 3a), although variations in the remodelling of microfilaments were apparent. Some cells formed actin spikes only on one side or along the protoplasmic protrusions, while in about $50 \%$ of the transfected cells, the actin cytoskeleton was modified as strongly as in infected cells. Therefore, genes that induced actin rearrangement were expressed by the HindIII plasmid library.

Based on these results, a series of cotransfection experiments in which one fragment at a time was removed from the plasmid library was performed. Only when the HindIII-D fragment was omitted did none of the cells show actin rearrangement (Fig. 4). However, the transfection of the HindIII-D 


\begin{tabular}{r|c|c|c|c|c|c|c|c|c|c|c|c|c|c|c|c|c|}
$\begin{array}{c}\text { Cells with actin } \\
\text { rearrangement }\end{array}$ & 16 & 21 & 7 & 11 & 0 & 12 & 13 & 26 & 16 & 16 & 46 & 2 & 2 & 106 & 0 & 0 & 31 \\
\cline { 2 - 13 } Total & 28 & 40 & 21 & 17 & 59 & 46 & 79 & 69 & 49 & 38 & 121 & 105 & 71 & 211 & 50 & 79 & 52 \\
\hline
\end{tabular}

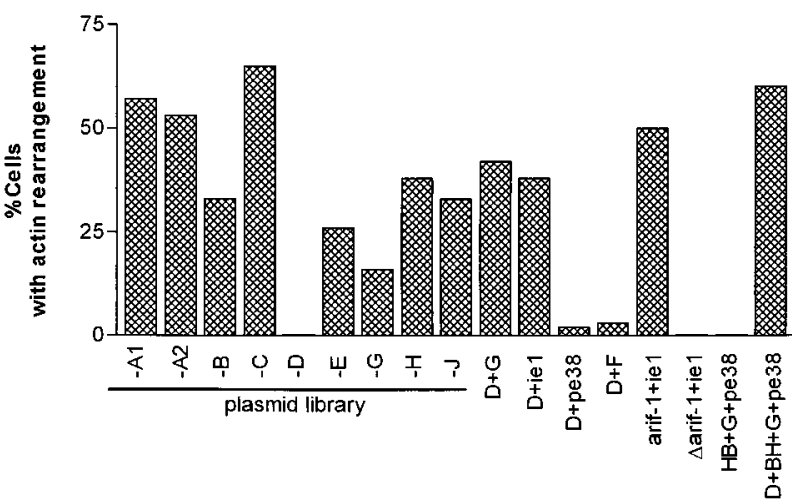

FIG. 4. Summary of transfection experiments. The total number of transfected cells derived from three or four independent experiments is compared to the number of cells with actin rearrangement. The percentage of cells with actin rearrangement is shown in a bar graph. The plasmid omitted from the plasmid library and the combinations of the transfected clones in the various experiments are indicated.

fragment alone was not sufficient for the remodelling of the actin cytoskeleton, indicating that additional factors are required. Therefore, cotransfection experiments were performed with plasmids expressing early genes, such as ie1 (14), ie2 (5), or pe38 (18), which have activating potential on viral promoters (21). The cotransfected plasmids were pHindIII-F encoding IE2 and PE38, pHindIII-G for IE0 and IE1, and the subclones pPE-HR1 and pClaI-ie1 expressing PE38 and IE1, respectively. Neither PE38 nor PE38 and IE2 supplied the putative stimulatory factor(s), although very few cells with actin rearrangement were observed (Fig. 4). In contrast, transfection of pHindIII-D together with IE1-expressing plasmids led to actin rearrangement in up to $40 \%$ of the transfected cells (Fig. 3b and 4), which is comparable to the transfection of the entire library. When IE1-expressing plasmids were transfected alone, actin rearrangement was never detected (Fig. 3e). Therefore, these results led to the notion that pHindIII-D provided the actin-rearrangement-inducing factor(s) while IE1 activated its gene expression. PE38 might have some very weak stimulatory effect on the expression of the putative actin-rearrangementinducing factor, since few cells with actin cables were detectable after cotransfection with pHindIII-D.

Identification of the arif-1 gene. To identify the gene encoding the actin-rearrangement-inducing factor, the HindIII-D fragment of about $9.6 \mathrm{kbp}$ was subcloned as detailed in Fig. 2. Transfection of the subclone $\mathrm{pBH} 3.7$ with IE1-expressing plasmids resulted in actin rearrangement. In contrast, transfection of the two subclones pPst-O and pBP2.1 of pBH3.7 (Fig. 2) did not lead to changes in the actin cytoskeleton (Fig. 3c and d). Therefore, these results suggest that the ORF disrupted in the two plasmids contributed to the induction of actin rearrangement. The disrupted ORF corresponds to the computer-predicted ORF21 in the published sequence of AcMNPV (1). By PCR amplification we have cloned ORF21 with $287 \mathrm{nt}$ of flanking sequences at the $5^{\prime}$ end and 502 nt at the $3^{\prime}$ end. Since transfection of the cloned ORF21 with the IE1-expressing plasmid induced actin rearrangement (Fig. 3f and 4), ORF21 was designated arif-1 (for actin-rearrangement-inducing factor 1). To further demonstrate that ARIF-1 indeed is the essential factor that causes actin rearrangement, the deleted version of the arif-1 ORF, p $\Delta$ arif-1 (Fig. 2), was cotransfected. None of the transfected cells showed rearrangement of the actin cytoskeleton (Fig. 4).

The function of IE1 as a transactivator of the arif-1 promoter was demonstrated by cloning the arif- 1 ORF under the control of the pe38 promoter, which is known to be regulated exclusively by host factors (18). In addition to the pe38/arif-1 cassette, the resulting plasmid pFHC-PE38/ARIF-1 contains the $i e 2$ gene, which serves as a marker to detect transfected cells by staining with a polyclonal antibody against IE2. After transfection of plasmid pFHC-PE38/ARIF-1, actin rearrangement occurred, indicating that IE1 is involved in the activation of the arif-1 promoter and is dispensable for actin rearrangement (Fig. 5).

Actin rearrangement was observed in only up to $60 \%$ of the transfected cells. These observations were made irrespective of whether the complete plasmid library, the two plasmids expressing ARIF-1 and IE1, respectively, or the pe38/arif-1 gene cassette was transfected. Therefore, we assume that the level of arif- 1 expression in each transfected cell might be important for the induction of actin rearrangement. Staining of transfected cells with the polyclonal antibodies against PE38 and IE2 revealed differences in the intensity of fluorescence, which strengthens the idea that the expression level of the transfected genes might be variable. Furthermore, we have to take into
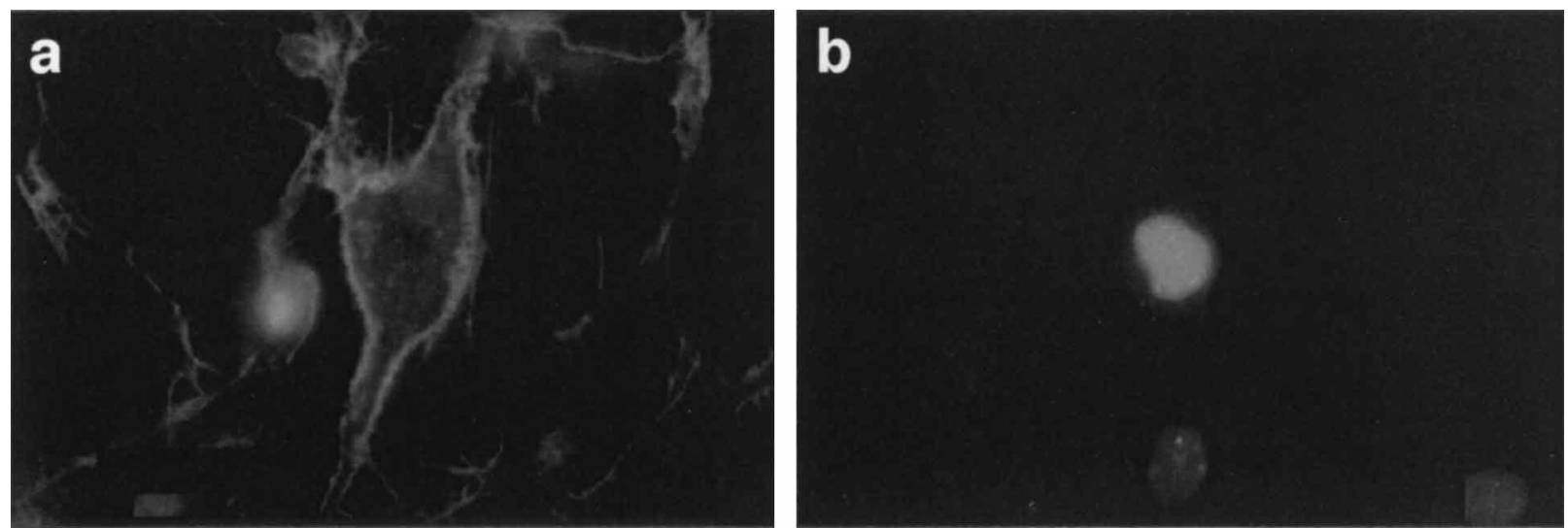

FIG. 5. Organization of the actin cytoskeleton after transfection of plasmid pFHC-PE38/ARIF-1. After transfection with pFHC-PE38/ARIF-1, TN-368 cells were processed as described in the legend to Fig. 1. After double labeling, the microfilaments (a) and staining with anti-IE2 antiserum (b) in the transfected cells are visible. 
1 GGCACCGACATGCATTTAATCG'TGTCARERPCCAACGTGCCGABFPIAGTATCAAACCTGATTGCGGGCAACGCTTCAATCGGCACGTAGTGCCITCTAC 101 GCATTACGGATTCAATGTAATCCATCCTATCGTCCAACGTGTCATIGTAATCTTGTTGGGCATGTCCGATATGCAAGTATGCCTGATAAAAGGGCTGGTA 201 CACTA $\underset{\text { PATAAA GAAACACGAACAGGAAAAACACGATCAGTACTCGATACATATTGCCATTACTTAGTTGTTGAATtAAATATTCAAAATG TTAAATAAAA }}{\stackrel{\Gamma}{\longrightarrow}}$ Psti

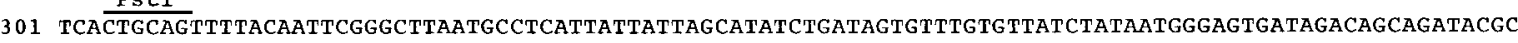
$\begin{array}{llllllllllllllllllllllllllllllllll}\text { T } & \text { A } & \text { V } & \text { L } & \text { Q } & \text { F } & \text { G } & \text { L } & \text { N } & \text { A } & \text { S } & \text { L } & \text { L } & \text { L } & \text { A } & \text { Y } & \text { L } & \text { I } & \text { V } & \text { F } & \text { V } & \text { L } & \text { S } & \text { I } & \text { M } & \text { G } & \text { V } & \text { I } & \text { D } & \text { S } & \text { R } & \text { Y } & \text { A }\end{array}$

401 GTTTTTGCTGGAAATTGAAGgAAAGCAATCGgTTATCAATCTATCTATACCGATAATGCIGGCGTTCGGCATGTGGATATTATTTTACATGTTTTATTTT

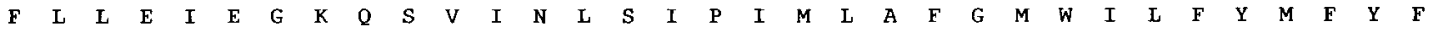

501 ATATGGAAGATTATCGTGTGGACGAAAAATCGCAGCAGCTCTTCAAACACAAATGTAAATTTCAATGCGAAAGAAAACTTTTACGTCGCTATCACGTGTA $\begin{array}{lllllllllllllllllllllllllllllllllll}I & W & K & I & I & V & W & T & K & N & R & S & S & S & S & N & T & N & V & N & F & N & A & K & E & N & F & Y & V & A & I & T & C & I\end{array}$

601 TTATGGTAAACGTGATCACTGGGCTGTGTTGGATGCTGTTTGCCGCGTTTCAAATCTACGTGTTCAAAAATGGTCATCTACCGGCGCTAGATGTTTTATA

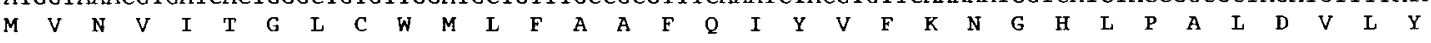

701 TCGTCACTACGATTTGGAATCAATGTGCTGGAACTCTATCGTGTATCTGGAAATTGACTACGCAAATACCGAAACCTTGAGCCAAAACTGTGTGTATGTG $\begin{array}{lllllllllllllllllllllllllllllllllll}R & H & Y & D & L & E & S & M & C & W & N & S & I & V & Y & L & E & I & D & Y & A & N & T & E & T & L & S & 0 & N & C & V & Y & V\end{array}$

801 AACATTTACAAAAAATGCATCATGTgCCGAGCGATTGTAAgCGATCACGAgCCGACCGTGTTTAATCAAAATTATCCCGTAATCATAATGGGCGTGCTTA

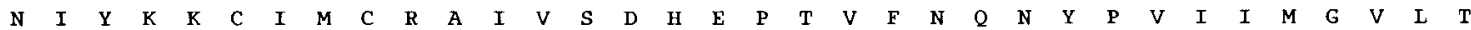

901 CGATACTGGCTGTGCAATGCTGGAATtTGTACGTGCAGTTAAAGGAGATGCGTCACAACATATACATGAAAAGGCGCGCCGAAGCCGAAAAGGCATCTTA

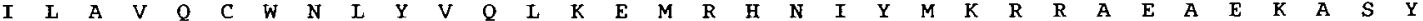
Xba I

1001 TGAACATTATTGCGACATTGACTATCGCCGCGAAGAACGAGAGAGTAACTCTAGATTGCTGGAAGTGGTCTCCGAAGGCAGAAATAGCAGCAGCGTGGTT

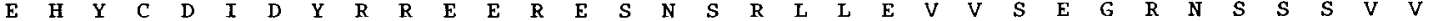

1101 GCCGTAACGCACCCGCCTTCTACCACTTCTACCACCACTTCGGTTTCAGAAACACTTTCATCTTTTATCGCGCCTTCGGACTTGTCTTCACAGCCATCGC

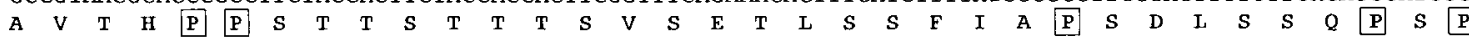

1201 CGTCTTCACACCCATCGTCTCCGTTCGGCAACCACAACGAGTTCTATTTTGGCAACACATATAGCGTGCCCAAGCCGGTGTACAGCGTGCCCAAGAAAGT

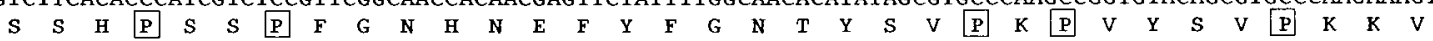

1301 TTTGTGTACCTCACCGGACGCGTGCACAATGTGCGAGCCTCCGTTGCCG GATGAAGAAATCATTAGTCGGCGCACGCCGACGCTTTCGCCCAAGCTGTTC

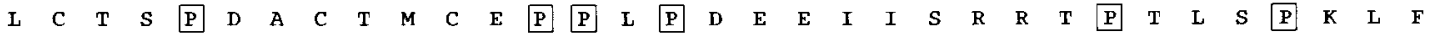

1401 CGCAAGTCCAGAGAACTGTCGCCCATCAAACCCGTAAGGACTCCTACTCCACCCGCTCCCACTCCACCGCCCATGTGTTTTAGCGAAGAGTTGCAGCGTA

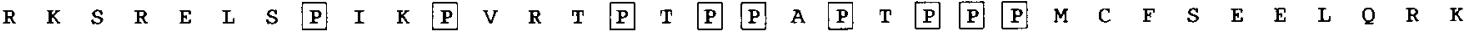

1501 AATTTCAAGAGAAAAAATATTACCCGTTTATGATGATTAGAAAGTATTATATAAAGTATTATTTTTGATTATATAAAGTACTAATTTAGTATTAGCGTA $\begin{array}{llllllllllllllllll}F & Q & E & K & K & \text { L } & \text { L } & \text { P } & \text { V } & \text { Y } & \text { D } & \text { D } & \star\end{array}$ 1601 ATTTAGTATTAATTTAGTACTAATgta polyA $\frac{\text { polyA }}{\text { polyA }}$

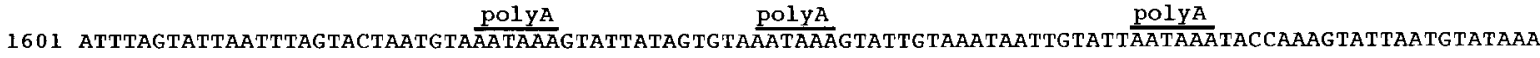

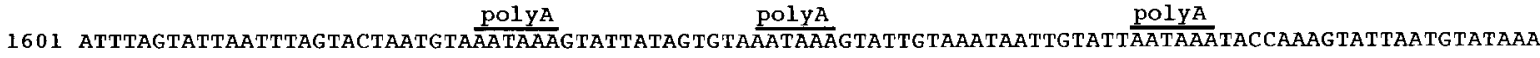
1701 TAAAATtgtatTATtTTAtaAaAaAtaAaAcaAtaAtTagaActgaAcaAg

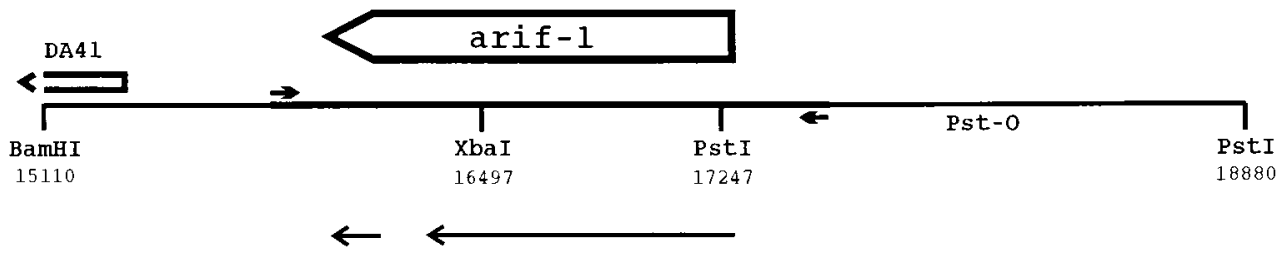

FIG. 6. Nucleotide sequence of arif-1. The DNA sequence of 1,751 nt, which includes the arif-1 gene and flanking sequences, represents the PCR-amplified fragment in plasmid pARIF-1. The predicted amino acid sequence is shown underneath the DNA sequence. The $5^{\prime}$ end determined by primer extension analysis is indicated by an arrow, a putative TATA signal is boxed, and potential polyadenylation sites are overlined. A stretch of $13 \mathrm{nt}$ which was variable in different virus strains is also overlined. Proline residues at the $\mathrm{C}$ terminus are boxed. The genomic localization of the arif- 1 gene is depicted at the bottom. The small arrows above and below the line represent the primers which were used for PCR amplification. The predicted ORF21 and ORF20 (1) are indicated by arrows.

account the fact that the host response to viral infection differs significantly from the response to transfection, which in turn might provide a less suitable environment for the induction of actin rearrangement.

Nucleotide sequence of the arif-1 gene. Synthetic primers were used to sequence both strands of the arif- 1 fragment in pARIF-1 (Fig. 2). The sequence of 1,751 nt is shown in Fig. 6, opposite to its genomic orientation. Computer analyses of the nucleotide sequences revealed the arif-1 ORF, potentially en- coding a 417-amino-acid polypeptide with a predicted molecular mass of $45 \mathrm{kDa}$, which is located on the leftward-transcribed strand according to the conventional orientation of the AcMNPV genome (29).

From the AcMNPV sequence of strain C6 (1) two ORFs, ORF21 and ORF20, encoding 319 and 69 amino acids, respectively, are predicted to occur in the same region and orientation as the arif-1 ORF (Fig. 6), while Braunagel and colleagues (4) found ORF5, corresponding to ORF21, by analyzing the 
AcMNPV sequence of strain E2. Therefore, we compared the nucleotide sequences of strains C6 and E2 with those of the arif-1 ORF which originates from the sequence of strain $\mathrm{E}$ (28). In contrast to the sequence of strain E, the C6 and E2 sequences were shown to carry an additional cytosine residue which generates the stop codon for ORF21/5. The absence of this cytosine residue in the E sequence causes an extension of the product of the arif- 1 ORF of 98 amino acids. To investigate whether the genomic sequences of the various AcMNPV strains indeed differ in the presence of the stop codon, we have cloned the arif- 1 fragments of strains E2 and L1 by PCR amplification with the viral genomes of strains E2 and L1 as templates. The determination of the corresponding sequences indicated that the predicted stop codon of ORF21/5 was not present. Finally, we sequenced part of the plasmid Pst-G, which is derived from the genome of the C6 strain, and in this case we also could not detect the predicted stop codon.

Furthermore, the alignment of the arif-1-specific sequences from the different AcMNPV strains demonstrated nucleotide substitutions and deletions in a stretch of $13 \mathrm{nt}$ at the $\mathrm{C}$ termini of strains E2, L1, and C6 as compared to strain E (Fig. 6). These differences were not confirmed by sequencing the corresponding fragments derived from strains E2, L1, and C6. In summary, we conclude that the arif-1 ORF is conserved in the different AcMNPV strains. Furthermore, the arif-1 ORF of AcMNPV has $88 \%$ nucleotide sequence identity to an ORF in the genome of Bombyx mori NPV encoding 443 amino acids (GenBank accession no. L33180).

A computer search for amino acid sequence homology of ARIF-1 with known proteins showed no significant sequence homology with any other proteins in the SWISSPROT database. There is some evidence for a signal peptide at the $\mathrm{N}$ terminus, which was also predicted by Ayres and colleagues (1). Furthermore, the $\mathrm{C}$ terminus carries a proline-rich region (Fig. 6) with no strikingly repetitive character (35).

Transcriptional activity of the arif-1 gene during the course of infection. In order to determine the time course of arif-1 expression during AcMNPV infection, the 5' end of the arif-1 transcript was analyzed by primer extension. Cytoplasmic RNA was isolated from uninfected TN-368 cells or from cells at 1,2 , 4, 6, 12, 24, and $48 \mathrm{~h}$ p.i. and hybridized to the arif-1-specific primer (Fig. 7). The precise lengths of the extended products were determined on sequencing gels (data not shown). The extended fragment of $167 \mathrm{nt}$ indicated the $5^{\prime}$ initiation site 55 nt upstream of the predicted ATG start codon and 4 nt upstream of a CAGT sequence motif (Fig. 6). Early transcriptional initiation is often found at or close to CAGT sequences (3). The arif-1 transcript was weakly detectable at $2 \mathrm{~h}$ p.i., becoming more abundant at 4 and $6 \mathrm{~h}$ p.i., and was hardly visible at $12 \mathrm{~h}$ p.i. The comparison of arif- 1 and ie1 transcription revealed that iel RNA was present as early as $1 \mathrm{~h}$ p.i. and increased in abundance at 6 to $48 \mathrm{~h}$ p.i. The extended product of $150 \mathrm{nt}$ indicated the previously described transcriptional start site of the iel gene (15). In contrast, the extension product of $339 \mathrm{nt}$ which reflects transcriptional initiation of the spliced RNA ieO (8) was observed only until $12 \mathrm{~h}$ p.i.

As a control, the same RNA preparation was analyzed for the presence of pe38 transcripts (Fig. 7). The extended products of $148 \mathrm{nt}$ representing the pe38 initiation site were detectable from 1 to $6 \mathrm{~h}$ p.i. The level of pe $38 \mathrm{RNA}$ at $1 \mathrm{~h}$ p.i. did not further increase until $6 \mathrm{~h}$ p.i., which is in contrast to the case for the ie1 transcript. While ie0, pe38, and arif-1 transcripts decreased at $12 \mathrm{~h}$ p.i., ie1 RNA was still present at an increased level.

In contrast to the transcription in infected TN-368 cells, pe38 transcription decreases at about 48 h p.i. after infection of $S$.
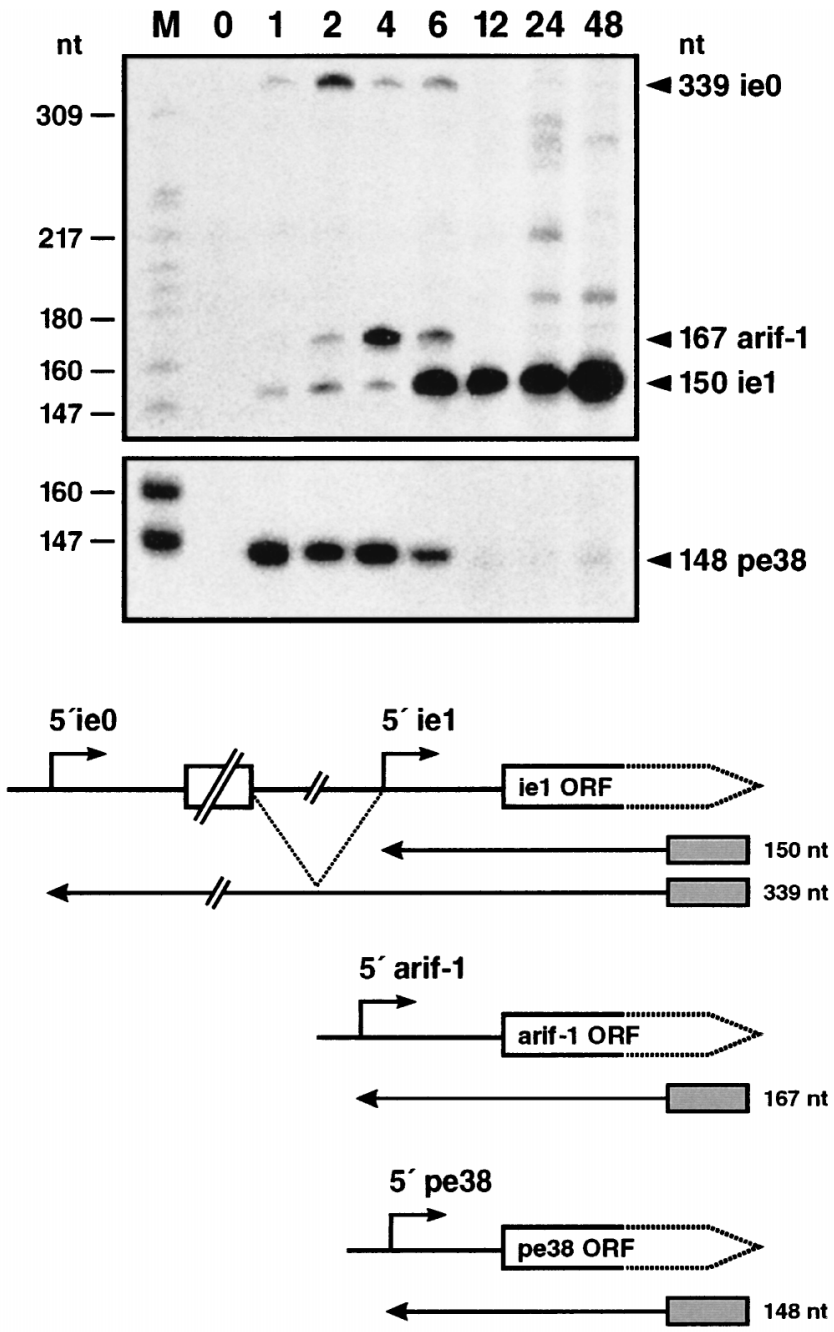

FIG. 7. Primer extension analysis of arif-1 transcription in the course of infection. Cytoplasmic RNA $(10 \mu \mathrm{g})$ prepared from uninfected TN-368 cells (lane 0 ) or at $1,2,4,6,12,24$, and $48 \mathrm{~h}$ p.i. (lanes $1,2,4,6,12,24$, and 48 , respectively) were hybridized to the arif-1- and ie $1 / \mathrm{ie} 0$-specific primers. The same RNA preparations were also hybridized to the pe38-specific primer. The extended products were analyzed on a $6 \%$ polyacrylamide gel and are depicted by arrows. Both autoradiograms were exposed overnight. Positions of DNA size markers are shown on the left (lane M). The localization of the extended products and the corresponding start sites are shown at the bottom. The intron of $i e 0$ is indicated by dotted lines.

frugiperda cells $(17,19)$. When infected $S$. frugiperda cells were analyzed for arif- 1 transcription by primer extension, we also observed the appearance of arif-1 RNAs until 24 to $48 \mathrm{~h}$ p.i. (data not shown), which might indicate that the infection cycle is extended in $S$. frugiperda cells as compared to TN-368 cells.

The results obtained with infected TN-368 cells demonstrate that arif- 1 RNA was present at $2 \mathrm{~h}$ p.i., suggesting a transcriptional delay of about $1 \mathrm{~h}$ when transcription is compared to that of $i e 0, i e 1$, and pe38. In contrast to ie1 transcripts, arif-1 RNAs were not detectable during the late phases of infection, which is comparable to the case for $i e 0$ and pe 38 transcripts.

Actin rearrangement in $\mathrm{TN}-368$ cells infected with the AcMNPV/Sf $M$ NPV hybrid. The presence of the arif- 1 transcript from 2 to $6 \mathrm{~h}$ p.i. corresponds to the accumulation of F-actin at the cell borders during infection. The causal link between arif- 1 expression and actin rearrangement in infected cells is sug- 

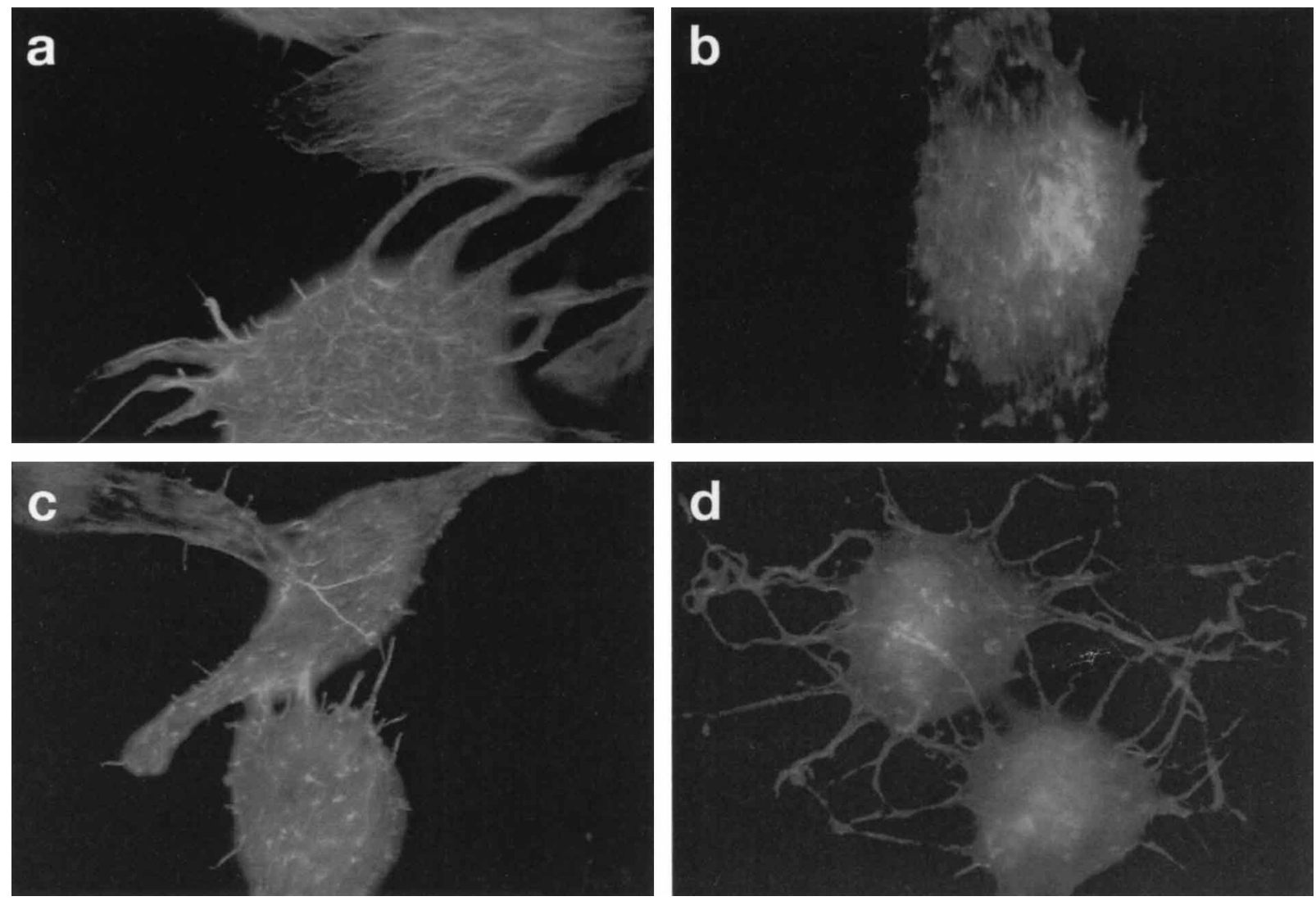

FIG. 8. Organization of the actin cytoskeleton in uninfected and AcMNPV/SfMNPV hybrid-infected TN-368 cells. Uninfected cells (a) or cells infected with the AcMNPV/SfMNPV hybrid (b to d) were fixed at 2 (b), 6 (c), and 16 (d) h p.i. The actin cytoskeleton was labeled with FITC-conjugated phalloidin.

gested by infection studies with the AcMNPV/S. frugiperda $M \mathrm{NPV}$ (AcMNPV/SfMNPV) hybrid (13). This mutant is viable in cell culture and contains the SfMNPV polyhedrin gene, which replaces the PstI O fragment of the AcMNPV genome, and it therefore carries a deletion in the arif-1 gene. After infection with the hybrid, actin rearrangement corresponding only to the initial induction of actin cables was observed, while the second stage of actin rearrangement was not detectable (Fig. 8b, c, and d). During the late phase of infection, nuclear F-actin was observed as in wild-type-infected cells, although a delay in appearance cannot be excluded. Additional recombinants containing only mutations in the arif- 1 gene need to be investigated to strengthen the results indicating that arif-1 expression causes the second step of actin rearrangement during the infection cycle. The functional significance has still to be elucidated. Since the deletion of the arif-1 gene in the AcMNPV/SfMNPV hybrid does not notably interfere with the production of viral progeny, it is tempting to speculate that the induced actin rearrangement is part of a host response which plays a significant role in the various tissues of the infected larvae.

In summary, we have identified the arif- 1 gene as a factor that is involved in the remodelling of the actin cytoskeleton during the early phase of AcMNPV infection. Actin rearrangement occurred after transfection of arif-1-expressing plasmids into TN-368 cells. The activation of the arif- 1 promoter most likely depends on the presence of IE1. Therefore, our studies provide the first insights into the functional role of actin rearrangement during the AcMNPV infection cycle.

\section{ACKNOWLEDGMENTS}

We thank Felicitas Jahnel for technical assistance, David O'Reilly for the gift of virus vEGTZ, Bob Possee for the gift of plasmid Pst-G, and Max Summers for kindly providing the AcMNPV/Sf $M$ NPV hybrid.

This research was supported by grant SFB-TP5 from the Deutsche Forschungsgemeinschaft and by the EC Biotechnology program BIOTECH (BIO2-CT94-3069).

\section{REFERENCES}

1. Ayres, M. D., S. C. Howard, J. Kuzio, M. Lopez-Ferber, and R. D. Possee. 1994. The complete DNA sequence of Autographa californica nuclear polyhedrosis virus. Virology 202:586-605.

2. Becker, D., and D. Knebel-Mörsdorf. 1993. Sequence and temporal appearance of the early transcribed baculovirus gene HE65. J. Virol. 67:5867-5872.

3. Blissard, G. W., and G. F. Rohrmann. 1990. Baculovirus diversity and molecular biology. Annu. Rev. Entomol. 35:127-155.

4. Braunagel, S. C., K. D. Daniel, L. M. Reilly, L. A. Guarino, T. Hong, and M. D. Summers. 1992. Sequence, genomic organization of the EcoRI-A fragment of Autographa californica nuclear polyhedrosis virus, and identification of a viral-encoded protein resembling the outer capsid protein VP8 of rotavirus. Virology 191:1003-1008.

5. Carson, D. D., L. A. Guarino, and M. D. Summers. 1988. Functional mapping of an AcNPV immediate early gene which augments expression of the IE-1 trans-activated 39K gene. Virology 162:444-451.

6. Charlton, C. A., and L. E. Volkman. 1991. Sequential rearrangement and nuclear polymerization of actin in baculovirus-infected Spodoptera frugiperda cells. J. Virol. 65:1219-1227.

7. Charlton, C. A., and L. E. Volkman. 1993. Penetration of Autographa californica nuclear polyhedrosis virus nucleocapsids into IPLB Sf 21 cells induces actin cable formation. Virology 197:245-254.

8. Chisholm, G. E., and D. J. Henner. 1988. Multiple early transcripts and splicing of the Autographa californica nuclear polyhedrosis virus IE-1 gene. J. Virol. 62:3193-3200.

9. Cudmore, S., P. Cossart, G. Griffiths, and M. Way. 1995. Actin-based mo- 
tility of vaccinia virus. Nature 378:636-638.

10. Cudmore, S., I. Reckmann, G. Griffiths, and M. Way. 1996. Vaccinia virus: a model system for actin-membrane interactions. J. Cell Sci. 109:1739-1747.

11. Danyluk, G. M., and J. E. Maruniak. 1987. In vivo and in vitro host range of Autographa californica nuclear polyhedrosis virus and Spodoptera frugiperda nuclear polyhedrosis virus. J. Invertebr. Pathol. 50:207-212.

12. Gardiner, G. R., and H. Stockdale. 1975. Two tissue culture media for production of lepidopteran cells and nuclear polyhedrosis viruses. J. Invertebr. Pathol. 25:363-370.

13. Gonzales, M. A., G. E. Smith, and M. D. Summers. 1989. Insertion of the SfMNPV polyhedrin gene into an AcMNPV polyhedrin deletion mutant during viral infection. Virology 170:160-175.

14. Guarino, L. A., and M. D. Summers. 1986. Functional mapping of a transacting gene required for expression of a baculovirus delayed-early gene. J. Virol. 57:563-571.

15. Guarino, L. A., and M. D. Summers. 1987. Nucleotide sequence and temporal expression of a baculovirus regulatory gene. J. Virol. 61:2091-2099.

16. Hink, W. F. 1970. Established insect cell line from the cabbage looper, Trichoplusia ni. Nature 226:466-467.

16a.Jahnel, F., and D. Knebel-Mörsdorf. Unpublished results.

17. Knebel-Mörsdorf, D., J. T. M. Flipsen, R. Roncarati, F. Jahnel, A. W. F. Kleefsman, and J. M. Vlak. 1996. Baculovirus infection of Spodoptera exigua larvae: lacZ expression driven by promoters of early genes pe 38 and me 53 in larval tissue. J. Gen. Virol. 77:815-824.

18. Krappa, R., and D. Knebel-Mörsdorf. 1991. Identification of the very early transcribed baculovirus gene PE38. J. Virol. 65:805-812.

18a.Krappa, R., and D. Knebel-Mörsdorf. Unpublished results.

19. Krappa, R., R. Roncarati, and D. Knebel-Mörsdorf. 1995. Expression of PE38 and IE2, viral members of the $\mathrm{C}_{3} \mathrm{HC}_{4}$ finger family, during baculovirus infection: PE38 and IE2 localize to distinct nuclear regions. J. Virol. 69: 5287-5293.

20. Lanier, L. M., J. M. Slack, and L. E. Volkman. 1996. Actin binding and proteolysis by the baculovirus AcMNPV: the role of virion-associated VCATH. Virology 216:380-388.

21. Lu, A., and E. B. Carstens. 1993. Immediate-early baculovirus genes trans- activate the p143 gene promoter of Autographa californica nuclear polyhedrosis virus. Virology 195:710-718.

22. Lynn, D. E., and W. F. Hink. 1980. Comparison of nuclear polyhedrosis virus replication in five lepidopteran cell lines. J. Invertebr. Pathol. 35:234-240.

23. Murges, D., A. Kremer, and D. Knebel-Mörsdorf. 1997. Baculovirus transactivator IE1 is functional in mammalian cells. J. Gen. Virol. 78:1507-1510.

24. Oppenheimer, D. I., and L. E. Volkman. 1995. Proteolysis of p6.9 induced by cytochalasin D in Autographa californica M nuclear polyhedrosis virus-infected cells. Virology 207:1-11.

25. Rice, W. C., and L. K. Miller. 1986. Baculovirus transcription in the presence of inhibitors and in nonpermissive Drosophila cells. Virus Res. 6:155-172.

26. Sanders, M. C., and J. A. Theriot. 1996. Tails from the hall of infection: actin-based motility of pathogens. Trends Microbiol. 4:211-213.

27. Sanger, F., S. Nicklen, and A. R. Coulson. 1977. DNA sequencing with chain-terminating inhibitors. Proc. Natl. Acad. Sci. USA 74:5463-5467.

28. Tjia, S. T., E. B. Carstens, and W. Doerfler. 1979. Infection of Spodoptera frugiperda cells with Autographa californica nuclear polyhedrosis virus. II. The viral DNA and the kinetics of its replication. Virology 99:399-409.

29. Vlak, J. M., and G. E. Smith. 1982. Orientation of the genome of Autographa californica nuclear polyhedrosis virus: a proposal. J. Virol. 41:1118-1121.

30. Volkman, L. E. 1988. Autographa californica MNPV nucleocapsid assembly: inhibition by cytochalasin D. Virology 163:547-553.

31. Volkman, L. E., and B. A. Keddie. 1990. Nuclear polyhedrosis virus pathogenesis. Semin. Virol. 1:249-256.

32. Volkman, L. E., P. A. Goldsmith, and R. T. Hess. 1987. Evidence for microfilament involvement in budded Autographa californica nuclear polyhedrosis virus production. Virology 156:32-39.

33. Volkman, L. E., and K. J. M. Zaal. 1990. Autographa californica M nuclear polyhedrosis virus: microtubules and replication. Virology 175:292-302.

34. Volkman, L. E., S. N. Talhouk, D. I. Oppenheimer, and C. A. Charlton. 1992. Nuclear F-actin: a functional component of baculovirus-infected lepidopteran cells? J. Cell Sci. 103:15-22.

35. Williamson, M. P. The structure and function of proline-rich regions in proteins. Biochem. J. 297:249-260. 